\title{
Análisis de la vía del parto en los diferentes escenarios asistenciales en Uruguay
}

\author{
Leonel Briozzo*, Giselle Tomasso ${ }^{\dagger}$, Valentina Colistro ${ }^{\ddagger}$
}

\section{Resumen}

Antecedentes: en Uruguay, el Ministerio de Salud ha seleccionado la disminución de las cesáreas innecesarias como uno de los objetivos sanitarios nacionales por considerarlo un problema de salud pública.

Objetivos: analizar las tasas de cesáreas por sector asistencial: público versus privado y Montevideo versus interior, usando la clasificación de Robson como insumo para su disminución.

Material y método: estudio descriptivo de corte transversal que categoriza cada nacimiento ocurrido en Uruguay en 2017 en uno de los diez grupos de Robson, según el sector de asistencia y el lugar geográfico donde ocurrió, informando el tamaño relativo, la tasa de cesáreas y las contribuciones absolutas y relativas hechas por cada grupo a la tasa global de cesáreas.

Resultados: la tasa de cesáreas en 2017 fue de 44\%. Los grupos que más contribuyeron a la tasa global de cesáreas (70\% de las cesáreas practicadas en el país) fueron: mujeres con una o más cesáreas previas, cesáreas electivas o inducciones fallidas en nulíparas y el grupo de nulíparas de término con inicio espontáneo. Luego de ajustar por las variables pronósticas, la probabilidad de terminar en cesáreas en el sector privado fue $18 \%$ mayor que en el sector público, sin mostrar diferencias en los resultados perinatales. Las tasas de cesáreas por grupos de Robson en Montevideo y en el interior se muestran muy similares.

Conclusiones: fomentar el parto vaginal después de una cesáreas, evitar cesáreas electivas e inducciones innecesarias y disminuir la primera cesáreas son las medidas propuestas para bajar las tasas de cesáreas innecesarias. Guías clínicas basadas en la mejor evidencia científica que sistematicen estas intervenciones ayudarán a implementar los cambios.

Palabras clave: Parto

Cesárea

Prevalencia

Clasificación de Robson

Servicios de salud

Key words: $\quad$ Parturition

Cesarean section

Prevalence

Robson classification

Health services

\footnotetext{
* Prof. Clínica Ginecotocológica A. Facultad de Medicina, Universidad de la República. Hospital de la Mujer, Centro Hospitalario Pereira Rossell. † Prof. Adj (I). Clínica Ginecotocológica A. Facultad de Medicina, Universidad de la República. Hospital de la Mujer, Centro Hospitalario Pereira Rossell.

$\ddagger$ Asistente Departamento de Métodos Cuantitativos. Facultad de Medicina, Universidad de la República.

Los autores declaran no tener conflicto de interés.

Este estudio no tiene financiación.

Correspondencia: Leonel Briozzo. Bvar. Artigas 1550. Montevideo, Uruguay. Correo electrónico: leobriozzo@hotmail.com

Recibido: 7/1/19

Aprobado: 26/2/19
} 


\section{Introducción}

En el último medio siglo la cesárea se ha difundido como vía de nacimiento y en la actualidad, desde la perspectiva de los cuidados de la salud, existe preocupación ya que la realización de una cesárea no justificada, lejos de mejorar, incrementa los riesgos maternos y perinatales. Este hecho ha transformando esta intervención en un problema ineludible de salud pública ${ }^{(1)}$. Así, se reporta un aumento de la morbimortalidad materna y perinatal cuando la prevalencia de cesáreas es mayor a $15 \%$ como vía de nacimiento ${ }^{(2)}$. Además, el aumento de las cesáreas se vincula con incrementos de las complicaciones en embarazos subsiguientes ${ }^{(3)}$.

A pesar de esta recomendación, en las últimas décadas se ha registrado un aumento progresivo en la proporción de cesáreas en la mayoría de los países, que en algunos casos supera el $90 \%$. Se estima que la tasa promedio mundial de cesáreas, según los últimos datos provenientes de 150 países ${ }^{(4)}$, es de $18,6 \%$ de todos los nacimientos, oscilando entre $7 \%$ y $40 \%$ en las regiones más y menos desarrolladas, respectivamente.

El incremento desmesurado de las cesáreas a nivel global y en nuestro país en particular (20\% en $1995 ; 31 \%$ en 2005; 44\% en 2014 y 2017) (figura 1), ha sido abordado institucionalmente por el Ministerio de Salud (MS), que ha seleccionado la disminución de las cesáreas innecesarias como uno de los objetivos sanitarios nacionales ${ }^{(5,6)}$. En este contexto, la Facultad de Medicina de la Universidad de la República (FMED-UDELAR), a iniciativa de la Clínica Ginecotocológica $\mathrm{A}^{(7)}$, ha conformado recientemente una comisión para elaborar una propuesta al respecto.

Se han planteado diferentes intervenciones tendientes a disminuir la tasa de cesáreas ${ }^{(8,9)}$. Una de las formas de abordar el problema es mediante la clasificación de Robson $^{(10)}$ recomendada por la Organización Mundial de la Salud (OMS) y por la Federación Internacional de Ginecología y Obstetricia (FIGO) como una forma sistematizada para evaluar, monitorear y comparar las tasas de cesáreas dentro y entre las instituciones de salud a lo largo del tiempo ${ }^{(11,12)}$. Este sistema, definido por el Dr. Michael Robson en $2001^{(13)}$, estratifica las mujeres según sus características obstétricas y, en consecuencia, permite comparar las tasas en cada grupo con un menor número de factores de confusión.

La clasificación (tabla 1) define diez grupos en base a cuatro conceptos obstétricos básicos: categoría del embarazo, antecedentes obstétricos, inicio del parto y edad gestacional, parámetros que permiten comparaciones en el tiempo de una misma unidad asistencial y entre distintas unidades. La clasificación de las mujeres en uno de los diez grupos permite identificar aquéllos que incrementan las tasas de cesáreas, reconociendo que unos son pasibles
Tabla 1. Clasificación de Robson

Grupo 1 Nulíparas con embarazo único en presentación cefálica, 37 semanas o más de gestación, trabajo de parto espontáneo.

Grupo 2 Nulíparas con embarazo único en presentación cefálica, 37 semanas o más de gestación, trabajo de parto inducido o parto por cesárea antes del comienzo del trabajo de parto.

Grupo 3 Multíparas sin cicatriz uterina previa, con embarazo único en presentación cefálica, 37 semanas o más de gestación, trabajo de parto espontáneo.

Grupo 4 Multíparas sin cicatriz uterina previa, con embarazo único en presentación cefálica, 37 semanas o más de gestación, trabajo de parto inducido o con parto por cesárea antes del comienzo del trabajo de parto.

Grupo 5 Multíparas con al menos una cicatriz uterina previa, embarazo único en presentación cefálica, 37 semanas o más de gestación.

Grupo 6 Nulípara con embarazo único en presentación podálica.

Grupo 7 Multíparas con embarazo único en presentación podálica, incluidas las mujeres con cicatrices uterinas previas.

Grupo 8 Mujeres con embarazos múltiples, incluidas las mujeres con cicatrices uterinas previas.

Grupo 9 Mujeres con embarazo único en situación transversa u oblicua, incluidas las mujeres con cicatrices uterinas previas.

Grupo 10 Mujeres con embarazo único en presentación cefálica, menos de 37 semanas de gestación, incluidas las mujeres con cicatrices uterinas previas.

de intervención y otros no. Asimismo, establece factores de riesgo modificables en estos grupos de pacientes, con lo cual se pueden implementar intervenciones focalizadas con mayor probabilidad de éxito para su disminución.

\section{Objetivo}

1. Identificar el perfil de la indicación de cesárea en Uruguay utilizando la clasificación de Robson comparando:

- los diferentes escenarios asistenciales: público o privado;

- el lugar geográfico de la maternidad donde ocurrió el nacimiento: Montevideo o interior.

2. Presentar recomendaciones en base a los hallazgos del estudio para cumplir con el objetivo ministerial de disminuir las cesáreas innecesarias. 


\section{Material y método}

Tipo y diseño de estudio: estudio descriptivo de corte transversal. Cada nacimiento de un recién nacido mayor de 22 semanas o de $500 \mathrm{~g}$ o más que tuvo lugar en Uruguay en el año 2017 fue clasificado según el lugar de nacimiento (Montevideo o interior) y el tipo de asistencia (subsector público o privado). Para esos cuatro grupos cada caso de cesárea fue clasificado a su vez en las diez categorías mutuamente excluyentes de Robson. Se comparó la proporción de cesáreas de esos diez grupos para los cuatro sectores (público vs privado y Montevideo vs interior). Se informó el tamaño relativo de cada grupo, la tasa en cada grupo y las contribuciones absolutas y relativas hechas por cada uno a la tasa global de cesáreas.

Material: para conocer la tasa de cesáreas se analizaron todos los nacimientos (mayor de 22 semanas o $\geq 500$ g) ocurridos en Uruguay en el año 2017, registrados a través del certificado electrónico de nacido vivo (CNV) que cubre el $100 \%$ de los nacimientos del país ${ }^{(14)}$.

Para poder desagregar las cesáreas en los grupos de Robson se utilizaron los nacimientos ocurridos en el país en 2017 que tuvieran registro en el Sistema Informático Perinatal (SIP) (o sea una historia clínica perinatal base [HCPB] almacenada en el software del SIP desarrollado por el Centro Latinoamericano de Perinatología [CLAP-OPS-OMS] cuyo uso es obligatorio por ley en Uruguay). La base SIP fue seleccionada por contar con todas las variables necesarias para el análisis de los nacimientos según la clasificación de Robson a diferencia del CNV.

Variables a medir: todas las necesarias para clasificar a las mujeres dentro de los diez grupos de Robson: paridad (nulípara, multípara), edad gestacional al momento del parto ( $\geq 37$ semanas, $<37$ semanas); presentación (cefálica, podálica, transversa/oblicua); multiplicidad de la gestación (único, múltiple); inicio del trabajo de parto (espontáneo, inducido, cesárea electiva) y antecedente de cesáreas. También se analizaron las variables lugar donde ocurrió el nacimiento, categorizando la información en Montevideo/interior, siendo Montevideo la capital y el interior el resto del país, y el efector público/privado según la modalidad de la asistencia. En Uruguay, según el informe del año $2017^{(15)}$, el $60 \%$ de las afiliaciones corresponden a coberturas asociadas al sector privado, ya sea que la persona posea derechos de atención en una de las 36 instituciones de asistencia médica colectiva (IAMC) o a través de uno de los seis seguros privados. La cobertura pública está representada por quienes se asisten en la Administración de los Servicios de Salud del Estado (ASSE), que concentra el 33\% de las afiliaciones, y por

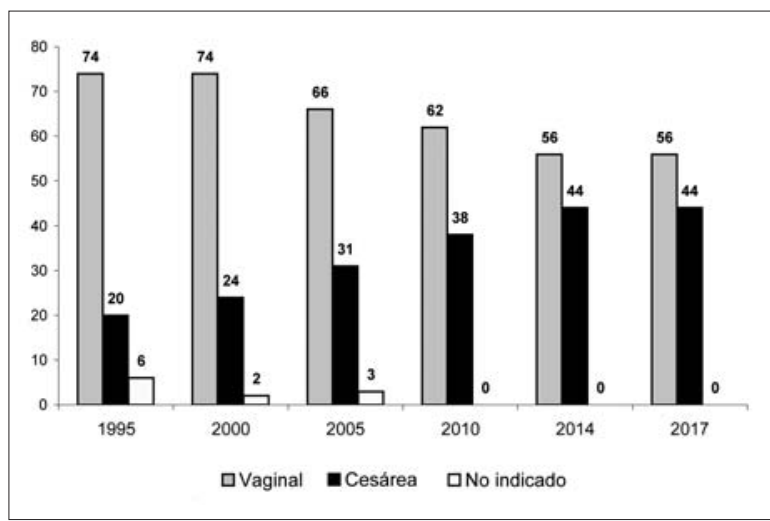

Figura 1. Evolución temporal del tipo de parto en Uruguay (porcentajes) 1995-2017.

las personas que tienen cobertura a través de Sanidad Militar o Sanidad Policial, que representan un 6\% del total de las afiliaciones.

Con el objetivo de evaluar la comparabilidad de las poblaciones (tabla 2), otras variables a medir fueron: edad materna $\geq 35$ años (sí/no); estado civil (soltera) (sí/no); duración de la educación igual a 6 años (sí/no); 0 gesta previa (sí/no); número de cesáreas previas (1, 2 o más); embarazo múltiple (sí/no); inicio de parto (inducido/cesárea electiva) (sí/no); presentación podálica (sí/no); otra presentación (oblicua/transversa) (sí/no); antecedente de diabetes (I, II, G) (sí/no); diabetes (sí/no); embarazo sin control (sí/no); partos asistidos por obstetras parteras (sí/no), factor reconocido como protector del parto vaginal ${ }^{(16)}$; porcentaje de falta de llenado en la variable cesárea anterior; peso al nacer al momento del parto (g) $(<1.500,1.500-2.499,2.500-3.999$, $\geq 4.000$ ); edad gestacional al parto (semanas) $<37$ semanas (pretérmino) $\geq 37$ semanas (término).

El porcentaje de falta de llenado de las variables seleccionadas (tabla3) se puede catalogar de muy bajo, lo que habla de una muy buena calidad de los datos, excepto para el antecedente de cesárea previa que mostraba una falta de registro de $10 \%(13,7 \%$ para el sector público y $7,8 \%$ para el sector privado).

Análisis estadístico. Los datos obtenidos se analizaron con Epi-Info ${ }^{\circledR}$ v 7.0 y con el software de análisis estadístico $\mathrm{R}^{(17)}$. Las diferencias y la significación estadística en los porcentajes de cesáreas fueron evaluadas utilizando la prueba de chi cuadrado y expresadas en RR y su índice de confianza (IC) a 95\%. La comparación de las variables pronósticas se realizó mediante una prueba de igualdad de proporciones y se reporta su significación estadística, usando 5\% como umbral. Para evaluar si la diferencia de cesáreas entre el sector público y el sector privado se mantiene al ajustar por las demás 
Tabla 2. Características individuales de las mujeres asistidas en sectores público y privado. Nacimientos Uruguay, 2017.

\begin{tabular}{|c|c|c|c|}
\hline Variable & $\begin{array}{c}\text { Sector público } \\
\begin{array}{c}N=15.648(39 \%) \\
\text { (sin dato\%) }\end{array}\end{array}$ & $\begin{array}{c}\text { Sector privado } \\
\begin{array}{c}N=25.016(61 \%) \\
\text { (sin dato\%) }\end{array}\end{array}$ & Valor $p$ \\
\hline Edad materna $>35$ años & $1.433(9)(0,2)$ & $5.126(20,5)(0)$ & $<0,00001$ \\
\hline Soltera & $4.046(26)(7)$ & $2.710(11)(0,9)$ & $<0,00001$ \\
\hline Duración de la educación (=6 años) & $4.819(31)(3)$ & $1.820(7)(0,8)$ & $<0,00001$ \\
\hline 0 gesta previa & $5.161(33)(0)$ & $9.592(38)(0)$ & $<0,00001$ \\
\hline 1 cesárea previa & $2.259(14)(9)$ & $4.654(18,6)(1)$ & $<0,00001$ \\
\hline 20 más cesáreas previas & $566(3,6)(9)$ & $860(3,4)(1)$ & 0,139 \\
\hline Embarazo múltiple & $324(2)(1)$ & $744(3)(0,3)$ & $<0,00001$ \\
\hline Inicio parto: cesárea electiva & $2.586(17)(5)$ & $6.766(27)(1,8)$ & $<0,00001$ \\
\hline Inicio parto: inducido & $2.427(15)(5)$ & $4.874(19,5)(1,8)$ & $<0,00001$ \\
\hline Prestación podálica & $497(3)(2)$ & $1.001(4)(1)$ & $<0,00001$ \\
\hline Otra presentación (oblicua/transversa) & $42(0,3)(2)$ & $105(0,4)(1)$ & 0,01094 \\
\hline Antecedente de diabetes $(I, I I, G)$ & $382(2,5)(2)$ & $787(3)(0,6)$ & $<0,00001$ \\
\hline Diabetes $(I, I I, G)$ & $2.092(13)(9)$ & $4.514(18)(0,8)$ & $<0,00001$ \\
\hline 0 control prenatal & $270(1,7)(1)$ & $154(0,6)(0,4)$ & $<0,00001$ \\
\hline Partos asistidos por parteras & $6.263(40)(2)$ & $2.307(9)(0,5)$ & $<0,00001$ \\
\hline Terminación en cesárea & $5.145(33)(0,7)$ & $12.910(51,6)(0,2)$ & $<0,00001$ \\
\hline$\%$ de falta variable cesárea anterior & $117(0,7)$ & $45(0,2)$ & $<0,00001$ \\
\hline \multicolumn{4}{|l|}{ Peso al nacer al momento del parto (g) } \\
\hline$<1.500$ & $249(1,6)(0)$ & $314(1,2)(0,7)$ & $<0,00001$ \\
\hline $1.500-2.499$ & $1.113(7)(0)$ & $1.619(6,4)(0,7)$ & 0,000287 \\
\hline $2.500-3.999$ & $13.096(84)(0)$ & $20.767(83)(0,7)$ & 0,02195 \\
\hline$>4.000$ & $1.164(7)(0)$ & $2.117(8,5)(0,7)$ & $<0,00001$ \\
\hline \multicolumn{4}{|l|}{ Edad gestacional al parto (semanas) } \\
\hline$<37$ (pretérmino) & $1.597(10)(2)$ & $2.473(10)(0,5)$ & 0,3983 \\
\hline$\geq 37$ (término) & $13.677(87)(2)$ & $22.323(89)(0,5)$ & $<0,00001$ \\
\hline Apgar $5^{\circ} \mathrm{min}:$ 0-6 & $273(1,7)(0,8)$ & $436(1,8)(0,4)$ & 0,9 \\
\hline
\end{tabular}

Fuente: SIP. Uruguay, Depto. Estadísticas vitales. Ministerio de Salud. 
Tabla 3. Falta de llenado de las variables de interés. SIP Uruguay, 2017. $\mathrm{N}=42.363$

\begin{tabular}{lcc|}
\hline \multirow{2}{*}{ Nombre de la variable } & \multicolumn{2}{c}{ Casos sin dato } \\
\cline { 2 - 3 } & $\mathrm{n}$ & $\%$ \\
\hline Gestas previas & 0 & 0 \\
Cesáreas previas & 1.862 & 4 \\
Inicio parto & 1.321 & 3 \\
Edad gestacional al parto & 502 & 1 \\
Presentación/situación & 629 & 1,5 \\
Embarazo múltiple & 325 & 0,7 \\
Vía terminación & 171 & 0,4 \\
\hline
\end{tabular}

variables pronósticas, se generó un modelo de regresión logística múltiple.

Aspectos éticos. Se mantuvo la confidencialidad de la información y el estudio fue aprobado por el Comité de Ética de la Facultad de Medicina y el Comité de Investigaciones del Centro Hospitalario Pereira Rossell. El uso de las bases de datos conteniendo la información del SIP del año 2017 fue autorizado por Estadísticas vitales del MS de Uruguay luego de cumplir con las solicitudes correspondientes. Las bases del SIP se recibieron anonimizadas, de acuerdo con la ley de protección de datos personales y porque para el presente trabajo no se requería identificación de las pacientes. Los datos del CNV fueron obtenidos del procesamiento en línea de los nacimientos del año 2017, disponible en la página web $\operatorname{del~} \mathrm{MS}^{(14)}$.

\section{Resultados}

En el año 2017, según el CNV ${ }^{(14)}$, se registraron 43.036 nacimientos en Uruguay; 26.219 en el sector privado, 16.650 en el sector público, 22.505 en Montevideo y 20.364 en el resto del país (167 sin datos). De esos nacimientos, $42.363(98,4 \%)$ cuentan con registro SIP y de ellos, 39.578 (92\%) tienen registradas todas las variables requeridas para la clasificación de Robson (figura 2).

La tasa global de cesáreas para el año 2017 fue de $44 \%$ (18.934/43.036): $51 \%$ en el sector privado (13.340) y $34 \%$ en el público (5.594). Las mujeres que se asistieron en el subsector privado presentaban mayor proporción de los siguientes factores de riesgo para cesárea comparadas con las del sector público (tabla 2): mayor edad, más años de educación, mayor porcentaje de casadas o concubinas, de nuliparidad y de diabetes, y menor participación de obstetras parteras en la atención del trabajo de parto y parto ( $9 \%$ en el sector privado vs $40 \%$ en el sector público).

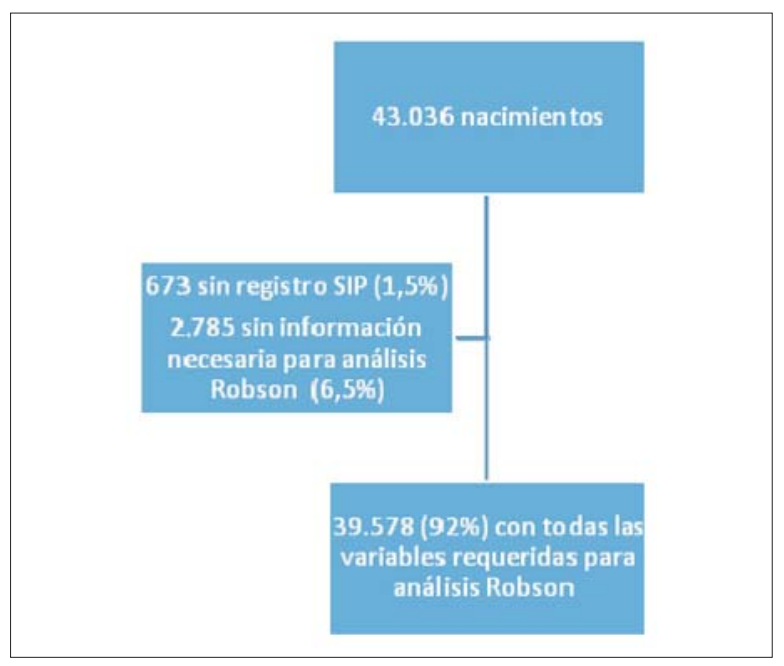

Figura 2.

La diferencia de cesáreas entre el sector público y el privado de casi 20 puntos porcentuales es estadísticamente significativa, $R R=1,51$ (IC95\%: 1,47-1,55) $(p<0,0001)$. $\mathrm{Al}$ ajustar en un modelo múltiple por las variables pronósticas que mostraban diferencias estadísticamente significativas entre los grupos comparados (tabla 2), la diferencia disminuye pero persiste y sigue siendo estadísticamente significativa. El valor de OR así ajustado es de 1,187 (IC95\%: 1,10-1,26), pudiendo decir entonces que una mujer asistida en el sector privado tiene $18,7 \%$ (10\%-26\%) más de probabilidad de terminar su embarazo por cesárea que si se asiste en el sector público y esa diferencia no se explica por ninguno de los factores pronósticos mal distribuidos que se muestran en la tabla 2.

El análisis de las cesáreas por subsector y lugar de atención del parto (tabla 4) mostró que las tasas de cesáreas son directamente proporcionales al número total de nacimientos asistidos.

Así, el sector privado asistió el $61 \%$ de los nacimientos y aportó el 70\% de las 18.934 cesáreas del país (Montevideo aportó $42 \%$ y el interior $29 \%$ ). Por su parte, el sector público, que asistió el 39\% de los nacimientos, aportó el $30 \%$ de las cesáreas, no existiendo grandes diferencias entre Montevideo e interior (13\% vs 17\% respectivamente) (tabla 5).

Independientemente del subsector público o privado, se observa que las tasas de cesáreas fueron mayores en Montevideo (55\%) que en el interior (45\%), siendo la diferencia estadísticamente significativa $R R=1,10$ (IC95\%: 1,08-1,12) (tabla 6).

La tabla 7 muestra la distribución de las cesáreas según los grupos de Robson para los sectores público y privado. Comparando el tamaño relativo de cada grupo 
Tabla 4. Vía del parto en diferentes escenarios asistenciales. Uruguay, 2017.

\begin{tabular}{|c|c|c|c|c|c|}
\hline \multirow[t]{2}{*}{ Gestión } & & \multicolumn{2}{|c|}{ Nacimientos totales } & \multicolumn{2}{|c|}{ Cesáreas } \\
\hline & & $F A$ & $F R$ & $F A$ & $F R$ \\
\hline \multirow[t]{2}{*}{ Privada } & Montevideo & 14.739 & $34 \%$ & 7.936 & $54 \%$ \\
\hline & Interior & 11.480 & $27 \%$ & 5.404 & $47 \%$ \\
\hline Subtotal & & 26.219 & $61 \%$ & 13.340 & $51 \%$ \\
\hline \multirow[t]{2}{*}{ Pública } & Montevideo & 7.766 & $18 \%$ & 2.459 & $32 \%$ \\
\hline & Interior & 8.884 & $21 \%$ & 3.135 & $35 \%$ \\
\hline Subtotal & & 16.650 & $39 \%$ & 5.594 & $34 \%$ \\
\hline Resto* & & 167 & $0 \%$ & 0 & $0 \%$ \\
\hline Total & & 43.036 & 100 & 18.934 & $44 \%$ \\
\hline
\end{tabular}

Tabla 5. Porcentaje de nacimientos y cesáreas aportadas por sector asistencial.

\begin{tabular}{llcc}
\hline Gestión & Departamento & Contribución $\%$ al total de nacimientos & Contribución \% al total de cesáreas \\
\hline Privada & Montevideo & $34 \%$ & $42 \%$ \\
& Interior & $27 \%$ & $29 \%$ \\
& Subtotal & $61 \%$ & $70 \%$ \\
\hline Pública & Montevideo & $18 \%$ & $13 \%$ \\
& Interior & $21 \%$ & $17 \%$ \\
\hline Total & Subtotal & $39 \%$ & $30 \%$ \\
\hline
\end{tabular}

Fuente: certificado de nacido vivo, Uruguay, 2017. Depto. Estadísticas vitales. Ministerio de Salud.

(columnas 6 y 7) se observa que $86 \%$ de las mujeres eran de los primeros cinco grupos, mientras que los grupos 6 , 7,8 y 9 solo representaron el $5 \%$ de los nacimientos para el sector público y $7 \%$ para el privado. Es interesante ver que el grupo 2 fue el que mostró mayores diferencias en cuanto a tamaño entre el sector público y el privado ( $10 \%$ público vs casi $20 \%$ en privado). Se trata de mujeres nulíparas con bajo riesgo de requerir una intervención y que, sin embargo, fueron sometidas a una inducción o a una cesárea de elección en mayor proporción en el sector privado que en el público. El sector público, por su parte (que asiste a la población más vulnerable), tuvo una mayor proporción de mujeres en el grupo 3 (multíparas, término, inicio espontáneo) $(28,5 \%$ vs $18,8 \%)$, explicable en parte porque la población de escasos recursos del país tiene tasas de fecundidad más elevada ${ }^{(18)}$. La proporción de mujeres en los otros grupos $(1,4,7,8$, 9 y 10) no mostró diferencias según que el subsector de atención fuera público o privado.

La tasa de cesáreas por grupo (columnas 8 y 9) fue siempre mayor en el sector privado que en el público. Las diferencias fueron estadísticamente significativas para todos los grupos excepto para el grupo 9 (situación transversa $u$ oblicua) $(\mathrm{p}=0,38)$.

Los grupos de Robson que mostraron más diferencias en la tasa de cesáreas entre el sector privado y el público fueron el grupo 10 (embarazo único, cefálica, pretérmino) (58\% en privado y $34 \%$ en público), el grupo 5 
Tabla 6. Vía del parto según lugar de nacimiento (Montevideo o interior).

\begin{tabular}{lcc}
\hline Lugar nacimiento & \multicolumn{2}{c}{ Tipo de parto } \\
\cline { 2 - 3 } & Vaginal & Cesárea (\%) \\
\hline Montevideo & 12.186 & $10.395(55)$ \\
Interior & 11.916 & $8.539(45)$ \\
Total & 24.102 & $18.934(100)$ \\
\hline
\end{tabular}

Fuente: certificado de nacido vivo. Estadísticas vitales. Ministerio de Salud. Uruguay, 2017. (mujeres con una o más cesáreas previas) ( $85 \%$ privado vs $65 \%$ público) y el grupo 2 (nulíparas, cefálica, término, inicio inducido o cesárea electiva) ( $72 \%$ privado y $56 \%$ público) (tabla 7 ).

Analizando la contribución relativa de cada grupo a la tasa global de cesáreas (columnas 10 y 11) se observa que quienes más contribuyeron fueron el grupo 5 (mujeres con una o más cesáreas previas), el grupo 2 (nulíparas, único, cefálica, término, inicio inducido o cesárea electiva) y el grupo 1 (nulíparas, cefálica, término, inicio espontáneo) aportando el $60 \%$ y $70 \%$ del total de las cesáreas.

Las mayores tasas de cesáreas en el sector privado no se asociaron a mejores resultados perinatales. La depre-

Tabla 7. Grupos de Robson según prestador (público vs privado). Nacimientos con Registro en SIP. Uruguay, 2017.

\begin{tabular}{|c|c|c|c|c|c|c|c|c|c|c|}
\hline \multirow[t]{2}{*}{ Población obstétrica } & \multicolumn{2}{|c|}{$N^{0}$ cesáreas } & \multicolumn{2}{|c|}{$N^{0}$ nacimientos } & \multicolumn{2}{|c|}{$\begin{array}{l}\text { Tamaño relativo del } \\
\text { grupo \% (1) }\end{array}$} & \multicolumn{2}{|c|}{$\begin{array}{l}\text { Tasa de CS/ } \\
\text { grupo \% (2) }\end{array}$} & \multicolumn{2}{|c|}{$\begin{array}{l}\text { Contribución relativa } \\
\text { a la tasa global } \\
\text { de cesáreas \% (3) }\end{array}$} \\
\hline & Público & Privado & Público & Privado & Público & Privado & Público & Privado & Público & Privado \\
\hline $\begin{array}{l}1 \text { - Nulíparas, único, cefálica, } \geq 37 \text { semanas, } \\
\text { inicio espontáneo. }\end{array}$ & 603 & 1.842 & 2.998 & 5.379 & $22 \%$ & $22 \%$ & $20,10 \%$ & $34,2 \%$ & $13 \%$ & $15 \%$ \\
\hline $\begin{array}{l}2 \text { - Nulíparas, único, cefálica, } \geq 37 \text { semanas, } \\
\text { inicio inducido o cesárea electiva. }\end{array}$ & 758 & 3.085 & 1.356 & 4.310 & $10 \%$ & $17,7 \%$ & $56 \%$ & $71,6 \%$ & $17 \%$ & $24 \%$ \\
\hline $\begin{array}{l}3 \text { - Multíparas, s/cicatriz de cesárea, único, } \\
\text { cefálica, } \geq 37 \text { semanas, inicio espontáneo. }\end{array}$ & 208 & 389 & 3.930 & 4.566 & $28,5 \%$ & $18,8 \%$ & $5,30 \%$ & $8,5 \%$ & $5 \%$ & $3 \%$ \\
\hline $\begin{array}{l}4 \text { - Multíparas, s/cicatriz de cesárea, único, } \\
\text { cefálica, } \geq 37 \text { semanas, inicio inducido o } \\
\text { cesárea electiva. }\end{array}$ & 408 & 847 & 1.179 & 2.106 & $8,6 \%$ & $8,6 \%$ & $34,60 \%$ & $40,2 \%$ & $9 \%$ & $7 \%$ \\
\hline $\begin{array}{l}5 \text { - Todas las multíparas con al menos una } \\
\text { cesárea previa, único, cefálica, } \geq 37 \text { semanas. }\end{array}$ & 1.467 & 3.844 & 2.262 & 4.505 & $16,5 \%$ & $18,5 \%$ & $65 \%$ & $85,3 \%$ & $32 \%$ & $30 \%$ \\
\hline 6 - Todas las nulíparas, único, podálica. & 151 & 487 & 177 & 507 & $1,3 \%$ & $2,2 \%$ & $85,3 \%$ & $96,0 \%$ & $3 \%$ & $4 \%$ \\
\hline $\begin{array}{l}7 \text { - Todas las multíparas, único, podálica, } \\
\text { incluye mujeres con cesárea previa. }\end{array}$ & 188 & 332 & 210 & 351 & $1,5 \%$ & $1,4 \%$ & $89,5 \%$ & $94,6 \%$ & $4 \%$ & $3 \%$ \\
\hline $\begin{array}{l}8 \text { - Todas las mujeres con embarazo múltiple, } \\
\text { incluye mujeres con cesárea previa. }\end{array}$ & 284 & 719 & 324 & 744 & $2,4 \%$ & $3,1 \%$ & $87,7 \%$ & $96,6 \%$ & $6 \%$ & $6 \%$ \\
\hline $\begin{array}{l}9 \text { - Todas las mujeres, único, en transversa } \\
\text { u oblicua, incluye mujeres con cesárea } \\
\text { previa. }\end{array}$ & 27 & 73 & 31 & 79 & $0,2 \%$ & $0,3 \%$ & $87 \%$ & $92,4 \%$ & $1 \%$ & $1 \%$ \\
\hline $\begin{array}{l}10 \text { - Todas las mujeres, único, cefálica, } \geq 36 \\
\text { semanas, incluye mujeres con cesárea previa. }\end{array}$ & 425 & 1.041 & 1.252 & 1.804 & $9,0 \%$ & $7,4 \%$ & $34 \%$ & $57,7 \%$ & $9 \%$ & $8 \%$ \\
\hline Total de nacimientos (4) & 4.519 & 12.659 & 13.719 & 24.351 & $100 \%$ & $100 \%$ & $33 \%$ & $52 \%$ & $100 \%$ & $100 \%$ \\
\hline
\end{tabular}

(1) $\mathrm{N}^{\circ}$ de nacimientos en cada grupo/ $\mathrm{N}^{\circ}$ total de nacimientos

(2) $\mathrm{N}^{o}$ de cesáreas $/ \mathrm{N}^{o}$ de nacimientos en el mismo grupo de Robson. (3) $\mathrm{N}^{o}$ de cesáreas en el grupo/ $\mathrm{N}^{o}$ total de cesáreas.

(4) Total de nacimientos con todas las variables necesarias para clasificación de Robson.

Fuente: SIP. Depto. Estadísticas vitales. Ministerio de Salud, 2017. 
Tabla 8. Grupos de Robson según lugar de nacimiento (Montevideo vs interior). Nacimientos con Registro en SIP. Uruguay, 2017.

\begin{tabular}{|c|c|c|c|c|c|c|c|c|c|c|}
\hline \multirow{3}{*}{$\begin{array}{l}\text { Población obstétrica } \\
\\
1 \text { - Nulíparas, único, cefálica, } \\
\geq 37 \text { semanas, inicio } \\
\text { espontáneo. }\end{array}$} & \multicolumn{2}{|c|}{$N^{0}$ cesáreas } & \multicolumn{2}{|c|}{$N^{0}$ nacimientos } & \multicolumn{2}{|c|}{$\begin{array}{c}\text { Tamaño relativo del } \\
\text { grupo \% (1) }\end{array}$} & \multicolumn{2}{|c|}{$\begin{array}{c}\text { Tasa de cesáreas/ } \\
\text { grupo \% (2) }\end{array}$} & \multicolumn{2}{|c|}{$\begin{array}{c}\text { Contribución relativa } \\
\text { a la tasa global de } \\
\text { cesáreas \% (3) }\end{array}$} \\
\hline & Montevideo & nterior & Montevideo & Interior & Montevideo & Interior & Montevidec & Interior & Montevide & Interior \\
\hline & 1.293 & 1.923 & 4.403 & 6.786 & $21,0 \%$ & $21,5 \%$ & $29,4 \%$ & $28,3 \%$ & $13 \%$ & $14 \%$ \\
\hline $\begin{array}{l}2 \text { - Nulíparas, único, cefálica, } \\
\geq 37 \text { semanas, inicio inducido } \\
\text { o cesárea electiva. }\end{array}$ & 2.425 & 3.222 & 3.678 & 4.890 & $17,6 \%$ & $15,5 \%$ & $65,9 \%$ & $65,9 \%$ & $25 \%$ & $23 \%$ \\
\hline $\begin{array}{l}3 \text { - Multíparas, s/cicatriz de } \\
\text { cesárea, único, cefálica, } \geq 37 \\
\text { semanas, inicio espontáneo. }\end{array}$ & 250 & 427 & 3.974 & 6.778 & $19,0 \%$ & $21,4 \%$ & $6,3 \%$ & $6,3 \%$ & $3 \%$ & $3 \%$ \\
\hline $\begin{array}{l}4 \text { - Multíparas, s/cicatriz de } \\
\text { cesárea, único, cefálica, } \\
\geq 37 \text { semanas, inicio } \\
\text { inducido o cesárea electiva. }\end{array}$ & 627 & 937 & 1.926 & 2.801 & $9,2 \%$ & $8,9 \%$ & $32,6 \%$ & $33,5 \%$ & $6 \%$ & $7 \%$ \\
\hline $\begin{array}{l}5 \text { - Todas las multíparas con } \\
\text { al menos una cesárea previa, } \\
\text { único, cefálica, } \geq 37 \\
\text { semanas. }\end{array}$ & 2.885 & 4.377 & 3.690 & 5.628 & $17,6 \%$ & $17,8 \%$ & $78,2 \%$ & $77,8 \%$ & $29 \%$ & $31 \%$ \\
\hline $\begin{array}{l}6 \text { - Todas las nulíparas, } \\
\text { único, podálica. }\end{array}$ & 398 & 544 & 426 & 583 & $2,0 \%$ & $1,8 \%$ & $93,4 \%$ & $93,3 \%$ & $4 \%$ & $4 \%$ \\
\hline $\begin{array}{l}7 \text { - Todas las multíparas, } \\
\text { único, podálica, incluye } \\
\text { mujeres con cesárea previa. }\end{array}$ & 304 & 450 & 326 & 486 & $1,6 \%$ & $1,5 \%$ & $93,3 \%$ & $92,6 \%$ & $3 \%$ & $3 \%$ \\
\hline $\begin{array}{l}8 \text { - Todas las mujeres con } \\
\text { embarazo múltiple, incluye } \\
\text { mujeres con cesárea previa. }\end{array}$ & 663 & 923 & 696 & 978 & $3,3 \%$ & $3,1 \%$ & $95,3 \%$ & $94,4 \%$ & $7 \%$ & $7 \%$ \\
\hline $\begin{array}{l}9 \text { - Todas las mujeres, único, } \\
\text { en transversa u oblicua, } \\
\text { incluye mujeres con cesárea } \\
\text { previa. }\end{array}$ & 61 & 87 & 67 & 96 & $0,3 \%$ & $0,3 \%$ & $91 \%$ & $90,6 \%$ & $1 \%$ & $1 \%$ \\
\hline $\begin{array}{l}10 \text { - Todas las mujeres, } \\
\text { único, cefálica, } \geq 36 \\
\text { semanas, incluye mujeres } \\
\text { con cesárea previa. }\end{array}$ & 885 & 1.256 & 1.754 & 2.599 & $8,4 \%$ & $8,2 \%$ & $50,5 \%$ & $48,3 \%$ & $9 \%$ & $9 \%$ \\
\hline Total de nacimientos (4) & 9.791 & 14.146 & 20.940 & 31.625 & $100 \%$ & $100 \%$ & $55 \%$ & $45 \%$ & 100 & $100 \%$ \\
\hline
\end{tabular}

sión neonatal (Apgar menor de 7 a los 5 minutos) no mostró diferencias entre ambos sectores (tabla 2).

Las tasas de cesáreas por grupos de Robson en Montevideo comparadas con el interior (tabla 8) se muestran muy similares excepto para los embarazos de pretérmino (grupo 10), que se operan más en Montevideo.

\section{Discusión}

Nuestros resultados coinciden con los hallazgos de estudios similares llevados a cabo en otros países. En Bra- $\mathrm{sil}^{(19)}$, con una tasa nacional de cesáreas de $52 \%$, los grupos de Robson que más contribuyeron fueron el 2, 5 y 10 , en ese orden. Otros tres estudios muestran tasas de $25 \%$ en Bosnia ${ }^{(20)}, 20 \%$ en Irán ${ }^{(21)}$ y $30 \%$ en Austra$\operatorname{lia}^{(22)}$. En los tres países, los grupos de Robson que más contribuyeron a la tasa global de cesáreas fueron también el 5, 2 y 1, al igual que en nuestro trabajo.

Limitaciones del estudio: para el análisis de las tasas de cesáreas, según los grupos de Robson, se debió recurrir a la base perinatal del SIP con 673 registros de naci- 
mientos menos que los ocurridos y registrados en Uruguay y con 2.785 registros SIP que no contaban con alguna de las variables necesarias para el análisis de Robson. De todas maneras, el $92 \%$ de los nacimientos (39.578/43.036) analizados contaban con muy buen nivel de llenado de las variables de interés y es poco probable que los registros faltantes estén asociados a dichas variables, por lo que se puede considerar un análisis no sesgado y representativo de la población del país.

El presente análisis pretende ayudar a los servicios de salud a identificar los grupos de mujeres que más contribuyen a las tasas generales de cesáreas, fomentar cambios en el manejo de esos grupos en la práctica clínica y disminuir las tasas innecesarias de cesáreas.

El análisis de los grupos de Robson comparando el sector público y el privado (tabla 7) ayuda a visualizar el diferente comportamiento profesional en la asistencia de mujeres con las mismas características, según el subsector de salud donde se trabaja. De ese análisis surge que la tasa de cesáreas fue mayor en el sector privado que en el público para todos los grupos y las mayores asimetrías se dieron en el manejo de los embarazos de pretérmino, de las mujeres con una o más cesáreas previas, en el manejo de las inducciones o cesáreas electivas en nulíparas, y del grupo 1 (nulíparas de inicio espontáneo). Estos cuatro grupos (5, 2, 1 y 10) fueron responsables del $80 \%$ de las cesáreas en el sector privado y de más del $70 \%$ en el sector público. Las acciones principales para lograr la disminución del número de cesáreas se deberán concentrar en esos grupos.

Algunas estrategias serían:

- Evitar la cesárea primaria considerando todos los esfuerzos para reducir las intervenciones obstétricas innecesarias y esperar el parto espontáneo.

- Revisar definiciones y tiempos de espera para diagnosticar trabajo de parto detenido, reduciendo el sobrediagnóstico de distocias.

- Mientras las condiciones maternas y fetales lo permitan, esperar los tiempos adecuados para las fases latentes y activas de la primera etapa y de la segunda etapa del trabajo de parto, que parecen ser más largas de lo que tradicionalmente se esperaba ${ }^{(23)}$.

- Limitar las inducciones/cesáreas electivas a lo estrictamente necesario.

- Ser estricto en la inducción del parto que debe realizarse solo por indicación médica; en caso de indicaciones no médicas, la edad gestacional debe ser de 39 o más semanas y el cuello uterino favorable (puntaje 8 de Bishop o mayor), especialmente en paciente nulíparas ${ }^{(24)}$.
- Facilitar el entrenamiento y la experiencia en el parto vaginal quirúrgico como método que bien indicado puede prevenir la cesárea con seguridad.

- Contar con guías clínicas y recomendaciones basadas en evidencias científicas que alienten y fomenten el uso seguro y apropiado del parto vaginal después de una cesárea. En la Maternidad del Centro Hospitalario Pereira Rossell ya se cuenta con guías clínicas publicadas que incluyen las recomendaciones antes sugeridas ${ }^{(25)}$.

- Educación y asesoría durante los controles obstétricos, explicando a las embarazadas y a sus parejas los riesgos a corto, mediano y largo plazo de las cesáreas, como la rotura uterina y las anomalías de la implantación placentaria, incluida la placenta previa y la placenta ácreta.

\section{Conclusiones}

1. La tasa de cesáreas se duplicó en Uruguay en los últimos 20 años, siendo de $44 \%$ para 2017.

2. Los grupos de Robson que más contribuyeron en la tasa de cesáreas, tanto en los sectores público como privado, fueron: el grupo 5 (cesárea previa), el grupo 2 (nulípara, término, cesárea electiva o después de una inducción fallida) y el grupo 1 (nulíparas, término, inicio espontáneo), representando más del $70 \%$ de las cesáreas practicadas en el país.

3. El subsector privado de atención presenta una mayor incidencia de cesáreas $(51 \%)$ que el sector público (34\%) y esa superioridad se explica en parte por las características de la población asistida en el sector privado con factores que aumentan el riesgo de cesárea, pero al ajustar por ellos la diferencia aún se mantiene con un riesgo 18\% mayor de cesáreas para el sector privado.

4. En Montevideo la tasa de cesáreas es similar a la del interior, salvo para el grupo de pretérminos (grupo 10) que se operan más en Montevideo posiblemente vinculado este aspecto a los traslados desde el interior a los centros de referencia ubicados mayormente en la capital.

5. Implicancias para la práctica. Si se pretende bajar las tasas de cesáreas es necesario implementar estrategias basadas en evidencias que impacten sobre los grupos 1 a 5 de Robson. Para eso, se necesita que los profesionales que tienen mayor tasa de cesáreas en nulíparas disminuyan su contribución con mejores decisiones en cada caso. Al evitar inducciones y cesáreas médicamente innecesarias (disminuir cesáreas en los grupos 1, 2, 3 y 4) y fomentar el uso seguro y apropiado del parto vaginal después de una cesárea (reducir el grupo 5) estaremos impactando en casi el $80 \%$ de todas las cesáreas. Sin olvidar que la 
estrategia del parto humanizado e institucional es la base para implementar cualquier estrategia de mejoramiento de la calidad de atención y seguridad en el área materno perinatal.

\section{Abstract}

Background: in Uruguay, the Ministry of Public Health has set the reduction of unnecessary C-sections as one of the "National Health Goals", as it considers this a public health issue.

Objectives:: to analyse the $\mathrm{C}$-section rates by health sector: the private sector versus the public sector and Montevideo versus the rest of the country, using the Robson classification and an input to be used to reduce the rate.

Method: transversal descriptive study that categorizes every birth in Uruguay during 2017 according to the Robson criterion in one of the 10 groups, depending on the healthcare center and the geographical place of birth, reporting the relative size, the $\mathrm{C}$-section rate and the absolute and relative contributions of each group to the global CS rate.

Results: in 2017 the CS rare was 44\%. The groups that contributed the most to the global CS rate $(70 \%$ of the CS in the country) were the following: women with previous CS, elective CS or failed induced labor in nulliparous women and the term nulliparous women with spontaneous initiation of labor group.

After adjusting the prognostic variables, the possibility of ending up in CS in the private sector was $18 \%$ higher than in the public sector, without there being any differences in terms of the perinatal results. CS rates by Robson group in Montevideo and the rest of the country are very similar.

Conclusions: vaginal birth must be encouraged after a CS, elective CS and unnecessary induction of labor must be avoided, and the number of first CS needs to be reduced. These are the measures proposed to reduce the unnecessary CS rates. Clinical guidelines based on the best scientific evidence that systematize these interventions will contribute to the implementation of changes.

\section{Resumo}

Antecedentes: o Ministério da Saúde do Uruguai (MS) definiu como um dos "Objetivos sanitários nacionais" a redução do número de cesáreas (CS) necessárias por considerar que este é um problema de saúde pública.

Objetivos: analisar as taxas de CS por setor assistencial: público vs privado e geográfico: Montevideo vs Interior usando a classificação de Robson, como insumo para sua redução.

Material e métodos: estudo descritivo transversal que classifica cada nascimento ocorrido no Uruguai em
2017, em um dos 10 grupos de Robson de acordo com o setor de assistência e o lugar onde aconteceu, informando tamanho relativo, taxa de CS e as contribuições absolutas e relativas de cada grupo à taxa global de CS.

Resultados: a taxa de CS em 2017, foi de $44 \%$. Os grupos que mais contribuíram para a taxa global de CS (70\% das CSs realizadas no país) foram: as cesareadas previas, as cesáreas eletivas ou induções falidas em nulíparas e o grupo de nulíparas a termo com início espontâneo. Depois de ajustar pelas variáveis prognósticas, a probabilidade de terminar em CS no setor privado foi $18 \%$ maior que no setor público, sem diferenças nos resultados perinatais. As taxas de CS por grupos de Robson em Montevideo e no Interior foram similares.

Conclusões: fomentar o parto vaginal depois de uma CS, evitar CS eletivas e induções desnecessárias e diminuir a quantidade de primeira CS, são las medidas propostas para reduzir as taxas de cesáreas desnecessárias. Pautas clínicas baseadas na melhor evidência científica que sistematizem estas intervenções ajudarão a implementar as mudanças propostas.

\section{Bibliografía}

1. Althabe F, Sosa C, Belizán J, Gibbons L, Jacquerioz F, Bergel E. Cesarean section rates and maternal and neonatal mortality in low-, medium-, and high-income countries: an ecological study. Birth 2006; 33(4):270-7.

2. Ye J, Betrán A, Guerrero Vela M, Souza J, Zhang J. Searching for the optimal rate of medically necessary cesarean delivery. Birth 2014; 41(3):237-44.

3. Schindl M, Birner P, Reingrabner M, Joura E, Husslein P, Langer M. Elective cesarean section vs. spontaneous delivery: a comparative study of birth experience. Acta Obstet Gynecol Scand 2003; 82(9):834-40.

4. Betrán A, Ye J, Moller A, Zhang J, Gülmezoglu A, Torloni M. The increasing trend in caesarean section rates: global, regional and national estimates: 1990-2014. PLoS One 2016; 11(2):e0148343. DOI: 10.1371/journal.pone.0148343.

5. Uruguay. Ministerio de Salud Pública. Documento técnico de maternidades. Montevideo: MSP, 2012. Disponible en: http://www.msp.gub.uy/sites/default/files/archivos_adjuntos/Documento $\% 20 t \% \mathrm{C} 3 \%$ A 9 cnico $\% 20 \mathrm{de} \% 20$ maternidades.pdf. [Consulta: 21 abril 2018].

6. Uruguay. Ministerio de Salud Pública. Presentación de Objetivos Sanitarios Nacionales 2020. Montevideo: MSp, 2015. Disponible en: http://www.msp.gub.uy/noticia/presentaci\%C3\%B3n-de-objetivos-sanitarios-nacionales-2020. [Consulta: 12 mayo 2018].

7. Uruguay. Universidad de la República. Consejo de la Facultad de Medicina. Exp. No 070011-001918-15. Montevideo, 28 de octubre de 2015.

8. Robson M, Scudamore I, Walsh S. Using the medical audit cycle to reduce cesarean section rates. Am J Obstet Gynecol 1996; 174(1 Pt 1):199-205. 
9. Chaillet N, Dubé E, Dugas M, Francoeur D, Dubé J, Gagnon $\mathbf{S}$, et al. Identifying barriers and facilitators towards implementing guidelines to reduce caesarean section rates in Quebec. Bull World Health Organ 2007; 85(10):791-7.

10. Robson M. Classification of caesarean sections. Fetal Matern Med Rev 2001; 12(1):23-39.

11. Betran A, Torloni M, Zhang J, Gülmezoglu A. WHO Statement on Caesarean Section Rates. BJOG 2016; 123(5):667-70. DOI: 10.1111/1471-0528.13526.

12. FIGO Working Group on challenges in care of mothers and infants during labour and delivery. Best practice advice on the 10-Group Classification System for cesarean deliveries. Int J Gynaecol Obstet 2016; 135(2):232-3.

13. Robson M, Hartigan L, Murphy M. Methods of achieving and maintaining an appropriate caesarean section rate. Best Pract Res Clin Obstet Gynaecol 2013; 27(2):297-308.

14. Uruguay. Ministerio de Salud Púbica. Departamento de Estadísticas Vitales. Estadísticas vitales: procesamiento de nacimientos. Montevideo: MSP, 2015. Disponible en: http://colo1.msp.gub.uy/redbin/RpWebEngine.exe/Portal?BASE=VITAL_NAC\&lang=esp. [Consulta: 1 agosto 2018].

15. Uruguay. Ministerio de Salud Pública. Área Economía de la Salud. Informe cobertura poblacional del SNIS según prestador 2017. Montevideo: MSP, 2017. Disponible en: https://www.gub.uy/ministerio-salud-publica/comunicacion/publicaciones/informe-cobertura-poblacional-delsnis-segun-prestador-2017. [Consulta: 12 mayo 2018].

16. Carlson N, Corwin E, Hernandez T, Holt E, Lowe N, Hurt K. Association between provider type and cesarean birth in healthy nulliparous laboring women: a retrospective cohort study. Birth 2018; 45(2):159-68.

17. R Foundation for Statistical Computing. R: A language and environment for statistical computing. Vienna, Austria: R CORE TEAM, 2017. Disponible en: https://www.r-project.org/. [Consulta: 12 mayo 2018].
18. Cabella W, coord. Análisis de situación en población Uruguay. Montevideo: UNFPA-Comisión Sectorial de Población, 2012

19. Nakamura-Pereira M, do Carmo Leal M, Esteves-Pereira A, Domingues R, Torres J, Dias M, et al. Use of Robson classification to assess cesarean section rate in Brazil: the role of source of payment for childbirth. Reprod Health 2016; 13(Suppl 3):128.

20. Fatusic J, Hudic I, Fatusic Z, Zildzic-Moralic A, Zivkovic M. Cesarean section rate analysis in University Hospital Tuzla - according to Robson's Classification. Med Arch 2016; 70(3):213-6.

21. Kazmi T, Saiseema S5th, Khan S. Analysis of cesarean section rate - according to Robson's 10-group Classification. Oman Med J 2012; 27(5):415-7.

22. Stavrou E, Ford J, Shand A, Morris J, Roberts C. Epidemiology and trends for caesarean section births in New South Wales, Australia: a population-based study. BMC Pregnancy Childbirth 2011; $11: 8$.

23. Zhang J, Landy H, Branch D, Burkman R, Haberman S, Gregory K, et al. Contemporary patterns of spontaneous labor with normal neonatal outcomes. Obstet Gynecol 2010; 116(6):1281-7.

24. Spong C, Berghella V, Wenstrom K, Mercer B, Saade G. Preventing the first cesarean delivery: summary of a joint $\mathrm{Eu}-$ nice Kennedy Shriver National Institute of Child Health and Human Development, Society for Maternal-Fetal Medicine, and American College of Obstetricians and Gynecologists Workshop. Obstet Gynecol 2012; 120(5):1181-93.

25. Centro Hospitalario Pereira Rossell. Hospital de la Mujer. Departamento Médico Obstétrico Maternidad "Augusto Tourenne". Protocolos clínicos en la maternidad, tomo 1: atención integral, humanizada e institucional de la mujer en trabajo de parto y parto y del recién nacido. Montevideo: UNFPA, 2017. 